\title{
Interactive comment on "Application of chemical derivatization techniques combined with chemical ionization mass spectrometry to detect stabilized Criegee intermediates and peroxy radicals in the gas phase" by Alexander Zaytsev et al.
}

Alexander Zaytsev et al.

zaytsev@g.harvard.edu

Received and published: 9 January 2021

Please find our response in the supplement.

Please also note the supplement to this comment:

https://amt.copernicus.org/preprints/amt-2020-335/amt-2020-335-AC1- 